Revista Herencia Vol. 27 (1 y 2), 47-56, 2014

Recibido 29-10-2013 Aprobado 16-11-2013

\title{
Proceso metodológico usado en el REGISTRO DE LAS GRÁFICAS RUPESTRES DEL Monumento Macional El Farallón (MNEF)
}

\begin{abstract}
RESUMEN
Este artículo detalla la metodología utilizada en el proceso de registro de las gráficas rupestres del Monumento Nacional El Farallón (MNEF), ubicado en Cedros de Cañas, como una alternativa viable que puede ser replicada en sitios patrimoniales de valor arqueológico. Además presenta un breve análisis de algunas de las gráficas rupestres que se encuentran en esa pared rocosa, asociadas a la fauna local.
\end{abstract}

Palabras claves: Monumento Nacional El Farallón, Región Arqueológica Gran Nicoya, gráficas rupestres.

\begin{abstract}
This article presents the methodology used in the process of registration of the El Farallón National Monument Rock Art, located in Cedros de Cañas, as a viable alternative that could be replicated in other archaeological sites. Futhermore, this article analyzes some rock art expressions associated to the local wildlife.
\end{abstract}

Keywords: El Farallón National Monument, Gran Nicoya Archaeological Region, rock art.

María Fernanda

Rojas. Estudiante de la Licenciatura en Antropología con énfasis en Arqueología, Universidad de Costa Rica. Estudiante de la Maestría Centroamericana en Gestión del Patrimonio Cultural para el Desarrollo, Universidad San Carlos de Guatemala. Licenciada en Ciencias de la Educación con énfasis en Administración de la Educación no Formal, Universidad de Costa Rica. Magíster en Gestión Ambiental y Ecoturismo. Bachiller en Antropología (con varios énfasis), Universidad de Costa Rica. arqueofer@hotmail.com

\section{Contextualización del MNEF}

El Monumento Nacional El Farallón (G-21 EF) es un sitio arqueológico que fue descubierto en 1987 por el Museo Nacional de Costa Rica en el poblado de Cedros de Cañas, en la provincia de Guanacaste, Costa Rica. Debido a su importancia para la comprensión de la historia antigua costarricense, el Ministerio de Cultura y Juventud lo declaró Monumento Nacional en 1998. Es también el inmueble arqueológico más grande que se conoce en el país, en propiedad privada, y el primer sitio arqueológico declarado monumento en la provincia de Guanacaste.

Aun así, este sitio ha sido escasamente investigado; los únicos registros de información existentes, antes de la presente investigación, son los informes técnicos del Ministerio de Cultura y Juventud (MCJ) y del Museo Nacional de Costa Rica (MNCR). (Duncan, 1998; Troyo, 1997; Troyo y Arguello, 2001; Sánchez, 2001; Valerio, 2001).

La particularidad de este sitio arqueológico reside en que allí existe una pared rocosa, la cual cuenta con 86 metros cuadrados de gráficas rupestres, 
creadas por sus primeros pobladores. La superficie de la pared rocosa es de 50 metros de largo por 30 metros de alto, de la cual 17,20 metros de largo y 5 metros de altura cuentan con gráficas rupestres antropomorfas, zoomorfas y abstractas, localizadas en la margen izquierda del río Cabuyo, a una altitud de 130 m.s.n.m. Según la inspección realizada por el MNCR en el MNEF, se encontró material cerámico del período Bagaces (300-800 d.C.), aunque de manera dispersa era "no diagnóstico" y estaba erosionado".

Dichas gráficas fueron realizadas mediante la técnica de la acanaladura y, de acuerdo con los datos geológicos, esta pared está formada por ignimbritas antiguas $^{2}$ (entre dos y cuatro millones de años) cubiertas por coladas de lava de 1-2 millones de años (Guillermo Alvarado, comunicación personal, 2006). Por otro lado, el contexto natural del sitio corresponde al bosque seco tropical transición a húmedo, caracterizado por una vegetación caducifolia y espinosa.

Según la arqueóloga E. Troyo (1997, sp.):

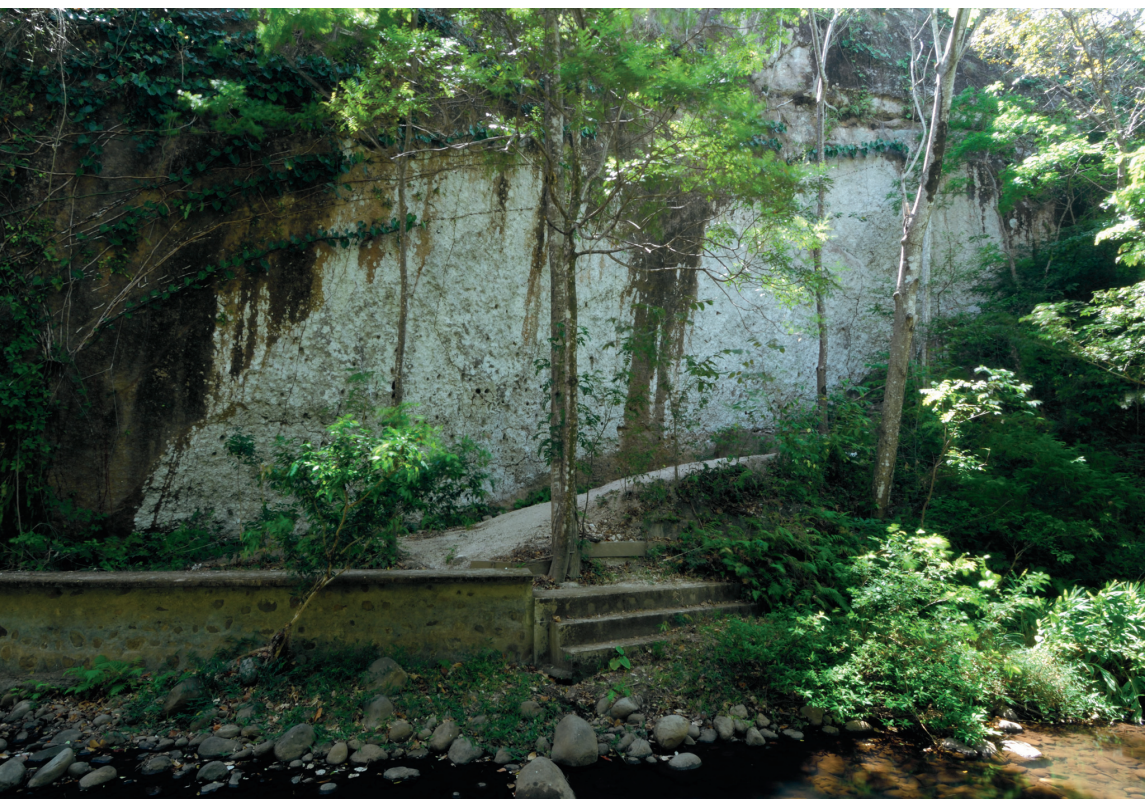

... Debe reiterarse que el Monumento Nacional El Farallón es uno de los pocos que existen a nivel nacional y que por su ubicación, dimensión y variedad de grabados puede interpretarse como un lugar de carácter ritual o ceremonial de las culturas indígenas.

\section{Metodología empleada en el registro de las gráficas rupestres}

La metodología usada en el MNEF respondió a la necesidad de buscar formas alternativas de trabajar en sitios de valor patrimonial de la mano

Figura 1. con las nuevas tecnologías para la recolección de la información arqueológica. Fotografía de pared rocosa donde se encuentran las gráficas rupestres del

MNEF.

Fuente: Fabio Hidalgo, 2010. En este caso, el registro de las gráficas rupestres resultaba infructuoso mediante las técnicas convencionales de la Arqueología como los calcos, el tizado y el dibujo. La primera técnica no era factible, dada la altura de la pared; la segunda, ya se encuentra en desuso pues causa alteraciones en las gráficas; y la última, por las dimensiones, la abundancia y el estado de estas -y por tener que recurrir a varios dibujantes, lo que podía repercutir en la calidad de las reproducciones de las gráficas y su posterior representación-, también se encuentra en desuso. Entonces, fue necesario utilizar una metodología de levantamiento de la información gráfica que permitiera trabajar sobre la pared rocosa sin alterar su estado de conservación.

Para tal efecto, se realizó una limpieza de la pared con agua desionizada. Seguidamente, se rellenaron los bajorrelieves de cada gráfica rupestre con algodón químicamente esterilizado y humedecido previamente con agua desionizada. Esto permitió redibujar las gráficas y lograr visualizar las imágenes en positivo (lo cual, con otras técnicas, se puede visualizar pero en negativo fotográfico). 


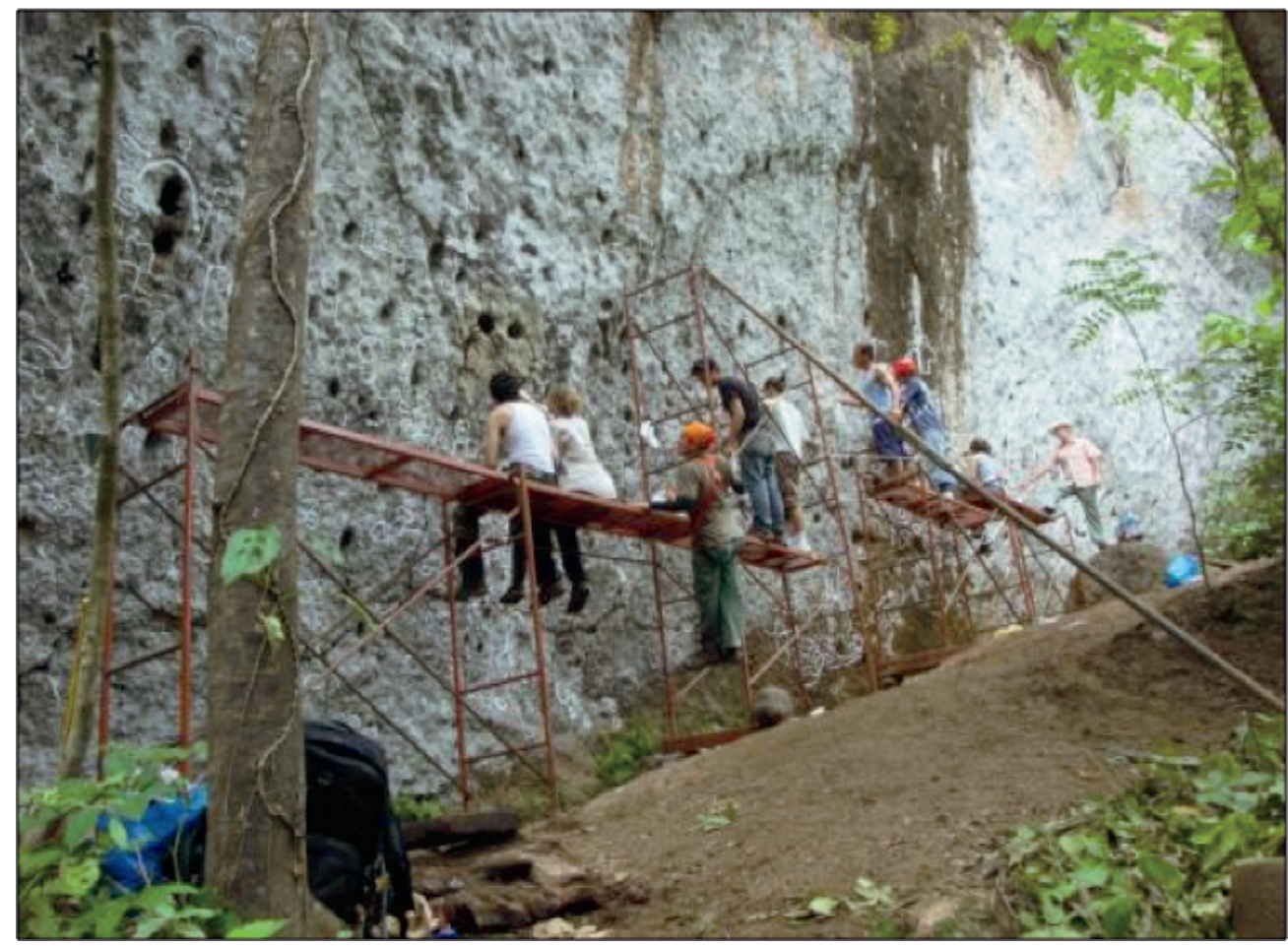

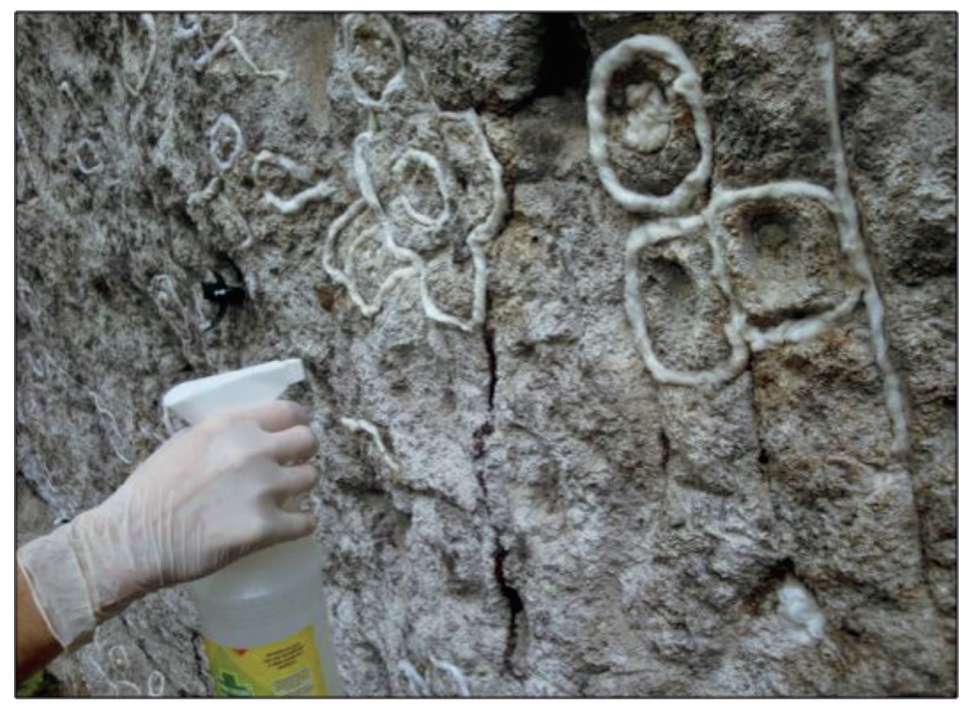

Figura 3. Limpieza de la pared rocosa. Fuente: William Monge, 2009.

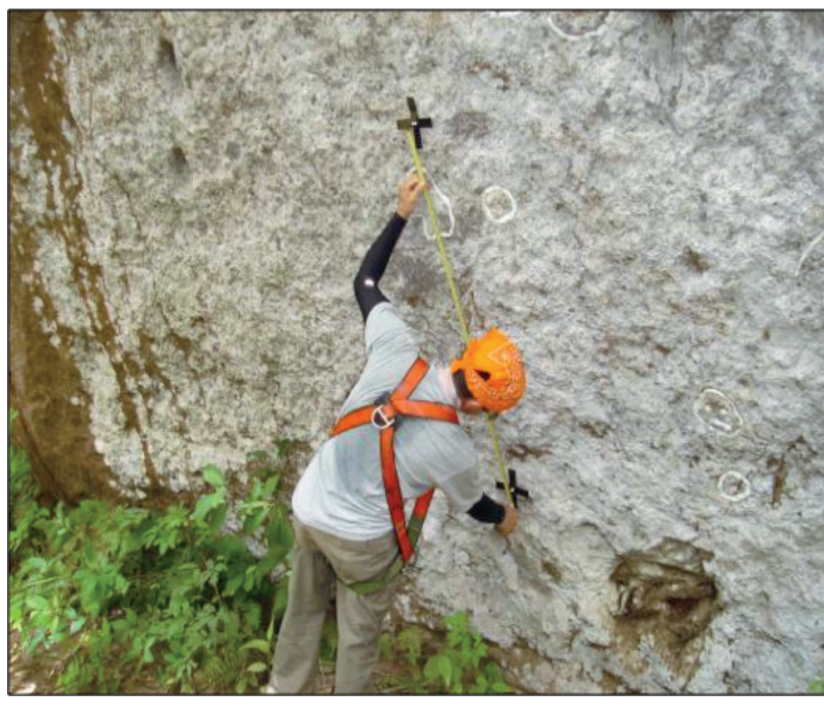

Figura 4. Montaje de las cuadrículas en la pared rocosa. Fuente: María Fernanda Rojas, 2009.

A continuación, se trazó una cuadrícula de 60 cuadros de 1,20 m por 1,70 m en la pared rocosa, donde se identificaron puntos nivelados topográficamente, para ejecutar así un proceso fotográfico minucioso de los 17,20 metros por 5 metros que están cubiertos por gráficas rupestres. Las fotografías se tomaron a 3 metros de distancia de esta pared, para mantener la escala y su posterior ensamblaje digital. 
Una vez tomadas las fotografías - mediante una cámara digital de alta resolución-, se trabajó con los programas Rhinoceros 4, Arc Map y Photoshop CS3 en su ensamblaje. Después se trasladó todo el trabajo previo de fotografía a Auto Cad 2009 para, así, dibujar, una a una, cada gráfica rupestre para su posterior análisis.

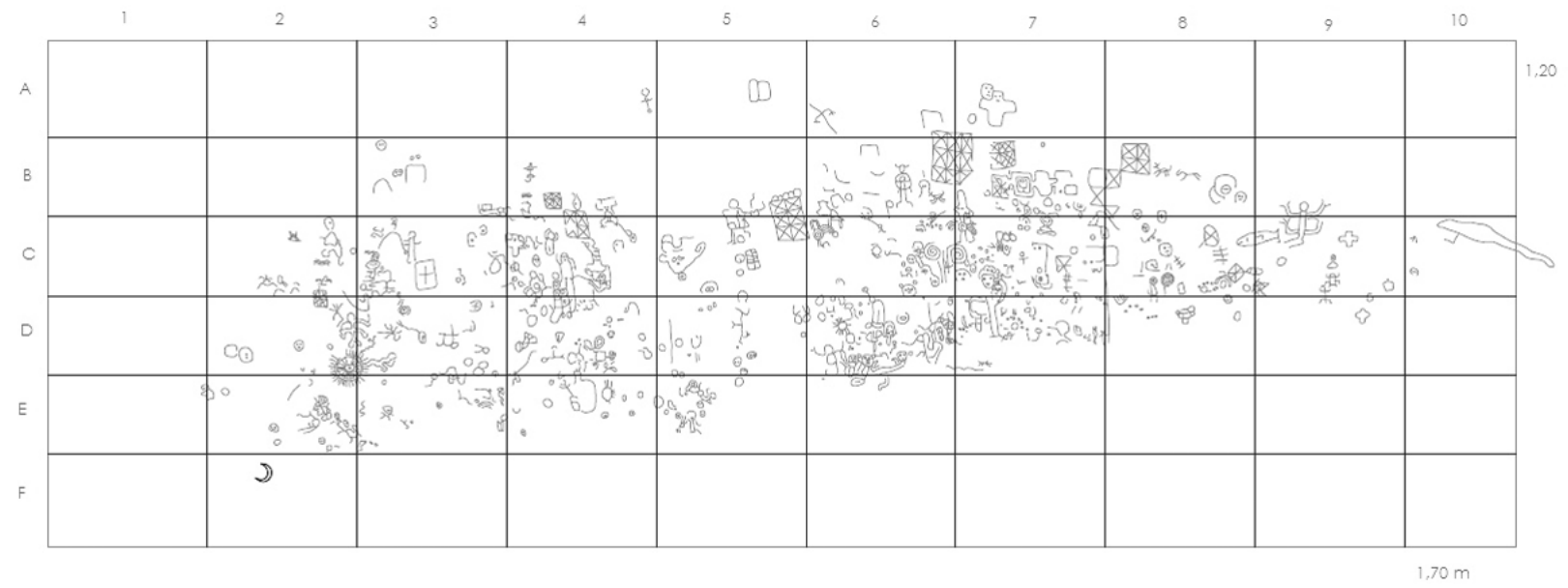

Figura 5. Plano cuadriculado con las gráficas rupestres del MNEF. Fuente: Elaboración propia con base en el dibujo: Adrián Bolaños, 2009.

Como se puede apreciar en el plano, la concentración de las gráficas rupestres se encuentra principalmente en las cuadrículas: 6D, 7B y 7C. Para efectos de esta investigación, se aclara que no se detalló el espesor de las acanaladuras de las gráficas ni se realizaron análisis estilísticos, ya que esto no está contemplado como un objetivo de esta investigación.

Un dato arqueológico importante de anotar fue la presencia de perforaciones bicónicas en la pared, las cuales son comunes en los sitios mayas y que, posiblemente, pudieron ser usadas para amarrar ciertos objetos y depositar allí flores u otros como símbolo de ofrendas a las deidades de la naturaleza (Gaspar Muñoz Cosme, comunicación personal, 2010). No obstante, estas oquedades pudieron tener también un uso funcional al ser utilizadas como parte de un sistema de andamiaje, debido a la altura de la pared, en el momento de grabar las gráficas. Estas evidencias son un material valioso por ser estudiado con detalle en el desarrollo de un posterior trabajo de investigación.

\section{Tipos de gráficas rupestres del MNEF}

Las gráficas rupestres que se encuentran en el MNEF son: antropomorfas ${ }^{3}$ (figuras humanas); abstractas, asociadas posiblemente a elementos simbólicos; y zoomorfas ${ }^{4}$ (figuras de animales), tanto en versión estilizada como naturalista. Estas últimas son las que se van a resaltar en este artículo.

En ese sentido, entre las figuras zoomorfas se encuentra una variada gama de animales propios del bosque seco tropical: saurios, serpientes, peces, aves, monos, felinos y otros mamíferos; ya que los indígenas precolombinos tenían 
un profundo conocimiento del bosque seco tropical y de lo que en él había. Sus necesidades básicas fueron satisfechas por la naturaleza. Por ejemplo, para el caso del MNEF, el pez identificado en una de las gráficas rupestres corresponde a un Machaca o Sabalete (Brycon guatemalensis), el cual habita los grandes lagos de Nicaragua y otros de Costa Rica, como el río Cabuyo, que pasa enfrente de la pared rocosa del MNEF. Este es un pez muy apetecido por su deliciosa carne, a pesar de tener muchas espinas. Este es un uso de la fauna con un carácter estrictamente alimenticio.

Por otra parte, algunos animales del bosque eran objeto de culto en las poblaciones precolombinas debido a ciertas características que poseían. En contextos arqueológicos funerarios es frecuente encontrar artefactos con representaciones de animales en materiales como oro, jade, piedra y cerámica. Los indígenas precolombinos les daban importancia a aquellos animales que se movían en más de un medio (agua, tierra, aire). Un animal que se desplazaba en dos medios era divinizado. De ahí que la serpiente, el jaguar y las aves, por ejemplo, ocupa-

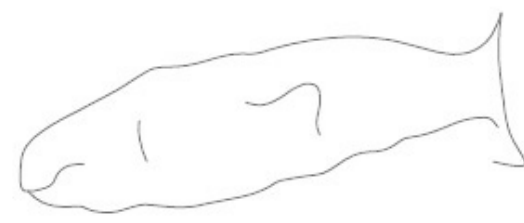

Figura 6.

Pez Machaca o Sabalete (Brycon guatemalensis) Fuente: Elaboración propia, 2009. ran un lugar especial en el imaginario mágico-religioso precolombino. Según Nicolás Caretta (2004) los mexicas y el resto de los pueblos no rinden culto a los animales per se, sino al valor simbólico que estos representan. Lo anterior no solo es aplicable a este grupo, sino a todos los pueblos precolombinos donde la fauna jugó un papel fundamental dentro de la cosmovisión indígena. Así, los dioses o héroes culturales que formaban parte de la ideología dominante entre los indígenas precolombinos de la Gran Nicoya estaban indiscutiblemente asociados a los animales de su entorno natural inmediato. Ejemplo de ello son tres de las gráficas plasmadas en el MNEF de carácter zoomorfo, interpretadas como las figuras de un felino, un saurio y un mono, representadas en versión naturalista.

En este sentido, los felinos fueron divinizados por sus atributos de fuerza, poderío y respeto, y además por ser excelentes cazadores en el bosque. En la tradición mesoamericana, el jaguar representa el elemento "tierra", y la serpiente, el elemento "agua", y la unión de ambos simbolizaría la fertilidad de la tierra por el agua. Esto es la unidad de la dualidad. Junto con el chompipe, el jaguar es el animal totémico del dios Tezcatlipoca. (Ferrero 2000, p.163).

Igualmente, según los mayas, a este animal se le liga con el sol nocturno del mito cosmogónico del Popol Vuh, llamado "Ixbalanqué" (Balam=jaguar), pues el sol se conviertía en jaguar al anochecer e ingresaba en el inframundo. Era la energía destructiva del mal, el dios de la muerte, el lado oscuro de la vida. Además representaba el alter ego de los personajes poderosos. De la misma manera, Snarskis, Salgado y Sánchez (2001, p. 85) señalan que "el jaguar y la serpiente emplumada, las principales deidades mesoamericanas sustituyeron al lagarto (cocodrilo) y al murciélago como figuras clave del contexto simbólico mitológico en la subregión Guanacaste-Nicoya".

Para el caso del saurio, en la tradición chibcha, "el cocodrilo es el que cumplía el papel del elemento agua como símbolo de la fertilidad" (Soto 2002, p. 22); 
Figura 8. Saurio, posiblemente de la familia Teiidae, género Ameiba. Fuente: Elaboración propia, 2009.

Figura 9. Mono, identificado como un mono araña o colorado (Ateles geoffroyi). Fuente: Elaboración propia, 2009. mas, en la ideología mesoamericana, específicamente en la mitología nahua, el cocodrilo o lagarto se relacionaba con el "Monstruo de la Tierra" (Tlaltecuhtli), en quien descansaba el mundo y, por ende, también los nacimientos y la vida (Ferrero 2000).

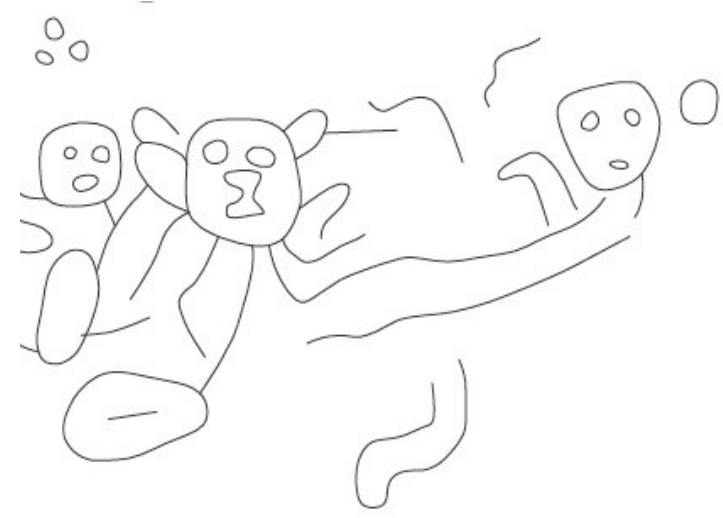

Figura 7.

Felino.

Fuente: Elaboración propia, 2009.

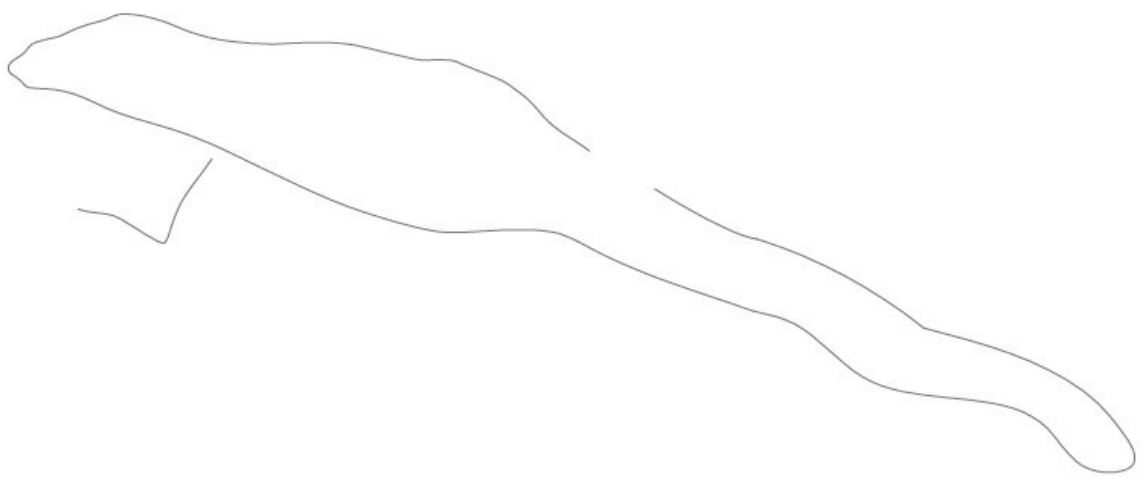

Por otro lado, el mono araña o colorado (Ateles geoffroyi) pudo ser admirado por

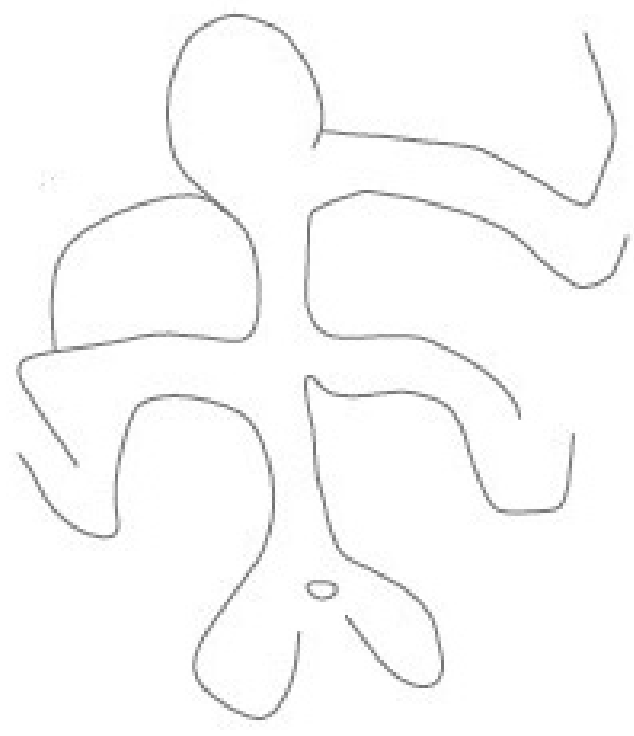
su agilidad y por la velocidad con que se mueve entre los árboles. Manzanilla (2006) lo asocia con el dios del viento Ehecatl-Quetzacóatl por su gran movilidad entre los árboles y su larga cola prensil que lo conectaba con las corrientes del aire y las nubes de lluvia. El mono también representa al dios de la danza, las canciones, la música, los juegos y la poesía: Xochpilli-Macoixochtl.

Finalmente, es importante destacar la relación "ser humano-naturaleza", que se visualiza en las gráficas rupestres. La fauna no sólo cumplió una función ecológica importante dentro 
del ecosistema del bosque seco tropical de Guanacaste, sino que también enmarcaba todos los aspectos de la vida cotidiana (alimento y vestido) y sagrada (religión, arte, creencias) de los indígenas precolombinos que habitaron esta región en tiempos antiguos. Sin embargo, a pesar de su singularidad, este sitio arqueológico actualmente experimenta un proceso de biodeterioro producto de la meteorización, además de otros problemas naturales que lo afectan como la escorrentía pluvial, los insectos y la vegetación. Lo más preocupante es que no existen mecanismos para proteger este patrimonio, de manera que se conserve como evidencia y marco de interpretación de nuestra historia antigua y de la región centroamericana.

\section{Notas}

1. De acuerdo con el informe emitido por la arqueóloga Elena Troyo, funcionaria del Centro de Investigación y Conservación del Patrimonio Cultural (CICPC) en 1997, las gráficas rupestres abarcan una extensión de 20 metros de largo por 7 metros de altura lo que $140 \mathrm{~m}^{2}$ de área con gráficas rupestres, medida que difiere con las tomadas en el trabajo de campo realizado para esta investigación.

2. La ignimbrita es un tipo de roca algo débil y fácilmente meteorizable y biodegradable.

3. Siguiendo las definiciones de Di Cosimo, las gráficas antropomorfas son las que presentan cuerpo, brazos, piernas y cara humana. Las gráficas zoomorfas muestran claramente características animales como patas, colas, pico, etc., y las gráficas abstractas son gráficas simbólicas o geométricas que presentan un grado de abstracción tal que su interpretación no conduce a nada de lo conocido en el mundo sensible (Di Cosimo, 1999).

4. Para el caso de las gráficas rupestres de índole zoomorfa se contó con la colaboración del biólogo Gino Biamonte Castro para la identificación del nombre científico de la fauna.

\section{Bibliografía}

Acuña Coto, Víctor. (1985). Un petroglifo de la cuenca media del Reventazón, Costa Rica: su análisis. San José: Departamento de Antropología, Universidad de Costa Rica.

Acuña Coto, Víctor y Óscar Fonseca Zamora. (Fall-Spring 1982-83). “Los petroglifos de Guayabo de Turrialba y su contexto". En: Journal of the Steward Anthropological Society. Vol. 14 (1-2).

Alcina Franch, José. (1999). "Los Aztecas". Biblioteca Historia 16. Madrid: Talleres Gráficos Peñalara S.A.

Alfaro Moisa, Claudia y Marielba Herrera Reina. (2008). "Sitio El Letrero: un espacio sagrado con grafías rupestres". Ponencia presentada en el IX Coloquio Guatemalteco de Arte Rupestre. Ciudad de Guatemala. 
Coe, Michael. (1975). Native Astronomy in Mesoamerica. Archaeoastronomy in Pre-Columbian America. Austin: University of Texas Press.

Coronado De Franco, Caterina y María Gabriela Arroyo Wong. (2002). Registro e interpretación de los petroglifos del Monumento Nacional El Farallón, provincia de Guanacaste. Propuesta de tesis en Arqueología. San José: Universidad de Costa Rica.

Di Cosimo, Patrizia. (1999). "Arte rupestre del sitio H y la Cueva del Murciélago, Archipiélago de Solentiname, Nicaragua". Revista Vínculos. Vol. 24 (1- 2): 2752. San José: Nuestra Tierra Editorial S.A.

Duncan Davis, Adolfo. (1998). Informe y recomendaciones para la conservación del petroglifo localizado en el sitio arqueológico Amazonas (Farallón). DPPC/ ASDD Farallón. San José: Departamento de Protección del Patrimonio Cultural, Museo Nacional de Costa Rica.

Fernández Esquivel, Patricia. (2008). Sellos precolombinos: imágenes estampadas de Costa Rica. San José: Fundación Museos Banco Central de Costa Rica. $1 .^{\circ}$ ed., segunda reimpresión.

Ferrero Acosta, Luis. (2000a). ¿Por qué prehistoria si hay historia precolombina? 2. ed. San José: EUNED.

Ferrero Acosta, Luis. (2000b). Costa Rica precolombina. 1. ed., sexta reimpresión. San José: Editorial Costa Rica.

Fonseca Zamora, Óscar. (2003). Historia antigua de Costa Rica: surgimiento y caracterización de la primera civilización. San José: Editorial de la Universidad de Costa Rica.

Fontana Coto, Amalia. (2001). Diseños del pasado que perduran para siempre: Hacia una aproximación en el diseño en el diseño bidimensional de la cerámica precolombina de la Región Arqueológica de la Gran Nicoya de Costa Rica. San José: INS.

Gelliot, Eric. (2006). "Investigando los petrograbados de Titihuapa (El Salvador): consideraciones metodológicas e interpretativas". Ponencia presentada en el VII Coloquio de Arte Rupestre. Ciudad de Guatemala.

Herrera Villalobos, Anayensy. (2005). Al reencuentro de nuestros ancestros. Lourdes de Montes de Oca: Editorial ICER.

Hurtado de Mendoza, Luis. (2004). Guayabo: historia antigua de Turrialba. San José: Litografía e Imprenta LIL S.A.

Ibarra Rojas, Eugenia. (2003). "Las sociedades cacicales de Costa Rica (siglo XVI)". Colección Historia de Costa Rica. 1. ${ }^{\circ}$ ed. Tercera reimpresión. San José: Editorial de la Universidad de Costa Rica. 
Künne, Martin. (2003). "Arte Rupestre de Costa Rica, Arte Rupestre de México Oriental y Centro América". Suplemento, 16: 163-181. Berlín: INDIANA.

Künne, Martin. (2007). "Los primeros viajeros europeos y las colecciones arqueológicas de Baja América Central". Istmo. Revista virtual de estudios literarios y culturas centroamericanas. N. ${ }^{\circ} 14$. Enero-junio.

Lerma Rodríguez, Félix Alejandro. (2009). "Las manos de la cueva del Ermitaño, El Salvador. Indagaciones espaciales en arte rupestre". Ponencia presentada en el $X$ Coloquio Guatemalteco de Arte Rupestre, Ciudad de Guatemala.

Manzanilla López Rubén. (2006). “Los petrograbados del sitio La Sabana, Acapulco Guerrero, México". Ponencia presentada en el VII Coloquio de Arte Rupestre. Ciudad de Guatemala.

Matilló Vila, Joaquín. (1973). Ometepe: Isla de círculos y espirales. Estudio del arte rupestre isleño. Volumen III. Managua: Publicaciones UCA.

Meighan, Clement W. (1995). "Central American rock art as viewed from Costa Rica. Rock art studies in the Americas". Oxbow Monograph 45. Editado por Jack Steinbring.

Mendiola Galván, Francisco (2002). Nuevas consideraciones en el estudio de la gráfica rupestre. Recuperado de: http://rupestreweb.tripod.com/mendiola.html

Monge-Nájera, Julián. (2004). Historia natural de Guanacaste. San José: EUNED.

Nicolás Caretta, Miguel. (2004). “La representación animal entre los mexicas: Una breve nota sobre el 'arte'". Ponencia presentada en el V Coloquio de Arte Rupestre, Ciudad de Guatemala.

Rivas Arévalo, Ernesto. (2010). "La Pintada: un sitio patrimonial en riesgo de desaparición". Kóot: Revista de museología. Año 1, diciembre, N.․ Museo Universitario de Antropología. San Salvador: Tecnoimpresos SA.

Sánchez González, Joaquín. (2001). Informe sobre la vegetación asociada al Monumento Nacional El Farallón, Cañas, Guanacaste. DHN-068-2001. San José: Departamento de Historia Natural, Museo Nacional de Costa Rica.

Snarskis, Michael Jay, Silvia Salgado y Luis Alberto Sánchez. (2001). Artes de América Central: en las colecciones del Museo Barbier- Mueller de Barcelona: Nicaragua, Costa Rica y Panamá. Barcelona: Institut de Cultura, Ajuntament e Barcelona.

Solís Del Vecchio, Felipe. (1996). Asentamientos y costumbres funerarias en la zona Cañas-Liberia durante el periodo Bagaces (300-800 d.C.). Tesis presentada para optar al grado de Licenciado en Antropología con énfasis en Arqueología. San José: Universidad de Costa Rica.

Soto Méndez, Zulay. (2002). Arte precolombino costarricense. San José: INS. 
Troyo Vargas, Elena. (1997). Propuesta de declaratoria Monumento Nacional El Farallón. DPH- 1112- 97. San José: Centro de Documentación del Patrimonio Cultural, Ministerio de Cultura, Juventud y Deportes.

Troyo, Elena y German Arguello. (2001). Diagnóstico del estado de conservación Monumento Nacional El Farallón, Guanacaste. San José: Centro de Investigación y Conservación del Patrimonio Cultural, Ministerio de Cultura, Juventud y Deportes.

Valerio Zamora, Ana Lucía. (2001). Informe geológico sobre el Monumento Nacional El Farallón. San José: Museo Nacional de Costa Rica.

Von Mayer, Peggy. (1989). "El sol en la mitología azteca". Káñina: revista de artes y letras de la Universidad de Costa Rica. Vol 13 (1-2): 157-163. 